\title{
Article \\ Influence of the Mixtures of Vegetable Oil and Vitamin E over the Microstructure and Rheology of Organogels
}

\author{
Renata Miliani Martinez ${ }^{1, *(D)}$, Pedro Leonidas Oseliero Filho ${ }^{2}$, Barbara Bianca Gerbelli ${ }^{3}$, \\ Wagner Vidal Magalhães ${ }^{4}$, Maria Valéria Robles Velasco ${ }^{1} \mathbb{D}$, Suzana Caetano da Silva Lannes ${ }^{5}$, \\ Cristiano Luis Pinto de Oliveira ${ }^{6}$, Catarina Rosado ${ }^{7}$ (D) and André Rolim Baby ${ }^{1, *(D)}$
}

1 Department of Pharmacy, Faculty of Pharmaceutical Sciences, University of São Paulo, São Paulo 05508-000, Brazil; mvrobles@usp.br

2 Department of Applied Physics, Physics Institute, University of São Paulo, São Paulo 05508-000, Brazil; plof@if.usp.br

3 Center of Natural and Human Sciences, Federal University of ABC, Santo André 09210-170, Brazil; barbara.gerbelli@ufabc.edu.br

4 Research \& Development Laboratory-Chemyunion Ltd., Sorocaba 18103-065, Brazil; wagner.magalhaes@chemyunion.com

5 Department of Biochemical and Pharmaceutical Technology, Faculty of Pharmaceutical Sciences, University of São Paulo, São Paulo 05508-000, Brazil; scslan@usp.br

6 Department of Experimental Physics, Physics Institute, University of São Paulo, São Paulo 05508-000, Brazil; crislpo@if.usp.br

7 CBIOS-Universidade Lusófona's Research Center for Biosciences and Health Technologies, 1749-024 Lisbon, Portugal; catarina.rosado@ulusofona.pt

* Correspondence: renata.martinez@usp.br (R.M.M.); andrerb@usp.br (A.R.B.)

\section{check for} updates

Citation: Martinez, R.M.; Oseliero Filho, P.L.; Gerbelli, B.B.; Magalhães, W.V.; Velasco, M.V.R.; da Silva Lannes, S.C.; de Oliveira, C.L.P.; Rosado, C.; Baby, A.R. Influence of the Mixtures of Vegetable Oil and Vitamin E over the Microstructure and Rheology of Organogels. Gels 2022, 8, 36. https://doi.org/ $10.3390 /$ gels 8010036

Received: 1 December 2021

Accepted: 2 January 2022

Published: 5 January 2022

Publisher's Note: MDPI stays neutral with regard to jurisdictional claims in published maps and institutional affiliations.

Copyright: (C) 2022 by the authors. Licensee MDPI, Basel, Switzerland. This article is an open access article distributed under the terms and conditions of the Creative Commons Attribution (CC BY) license (https:// creativecommons.org/licenses/by/ $4.0 /)$.

\begin{abstract}
Candelilla wax (CW) and 12-hydroxystearic acid (12HSA) are classic solid-fiber-matrix organogelators. Despite the high number of studies using those ingredients in oily systems, there is scarce literature using a mixture of oil and antioxidants. Vitamin E (VE) is an important candidate for its lipophilicity and several applications on pharmaceutical, cosmetics, and food industries. In this work, we investigated the influences of mixtures between vegetable oil (VO) and VE on the microstructures and rheological properties of $\mathrm{CW}$ and 12HSA organogels. A weak gel $\left(\mathrm{G}^{\prime \prime} / \mathrm{G}^{\prime}>0.1\right)$ with a shear-thinning behavior was observed for all samples. The presence of VE impacted the gel strength and the phase transition temperatures in a dose-dependent pattern. Larger and denser packed crystals were seen for 12HSA samples, while smaller and more dispersed structures were obtained for $\mathrm{CW}$ organogels. The results obtained in this work allowed the correlation of the structural and mechanical properties of the organogels, which plays an important role in the physical-chemical characteristics of these materials.
\end{abstract}

Keywords: organogel; candelilla wax; 12-hydroxystearic; vitamin E; biomaterials; rheology; SAXS

\section{Introduction}

Organogels are tridimensional structures formed by the organization of molecules in order to hold an organic solvent. Gel formation can be investigated by crystal network observation via polarized microscopy, rheological viscoelastic properties, and/or physical structure at the atomic scale via small-angle X-ray scattering (SAXS) [1]. The type of organogelator and the production process allow the classification of organogels into three categories: (a) fluid filled matrix; (b) solid fiber matrix; and (c) chemical organogels [1]. Low-molecular-weight organogelators (LMWOs), such as candelilla wax (CW) and 12hydroxystearic acid (12HSA), can self-organize within an organic system, forming a thermoreversible organogel [2]. The gelation occurs upon the total melting of organogelators, followed by a cooling process as the driving force for the nucleation of the organogelator 
molecules. This mechanism, known as solid fiber, leads to a three-dimensional fibrillar network structure that holds the organic phase [1].

The crystallization pattern (the shape, size, and distribution of crystals) of organogels is a manifestation of several interaction forces between organogelators and the organic solvent and is affected by many variables, such as the physical and chemical properties of the components, the organogelator/solvent ratio, the total melting of the organogelator, and the cooling rate [3]. The starting point for organogelification is the nuclei formation that grows into the crystalline network. Heterogeneous nucleation is the most favorable type of process, when the nuclei are formed by a substrate that provides the orientation for the crystalline network. The substrate can be any surface, such as the walls of the container or even impurities present in solutions [4]. Therefore, every organogel component is a potential nucleation agent, especially if it is solid or crystalline by itself. For that reason, most organogel studies are conducted with one simple organic phase for each organogelator, such as sunflower oil and CW [5,6] or canola oil and 12HSA [7]. However, for practical applications, more complex matrices are often used. For instance, when oil is used as the organic phase of organogels, the presence of a lipophilic antioxidant could protect against degradation. Despite robust information regarding the organogel's structure using mixed sterols [8] and lecithin blended with other materials [9], few information about mixtures with antioxidants is available.

Our research group has been studying the influence of antioxidants on colloidal systems for the past decade and has recently started exploring organogels and bigels with vitamin E (VE) [10]. Despite beneficial to humans, VE is not naturally produced by the human body and must be topically or orally delivered. This antioxidant is involved in cutaneous protective pathways against aging, atopic dermatitis, and melanoma, for instance [11-13]. It also plays an important role in food industry as a shelf-life enhancer of oily products or to increase oxidative stability in heated oils [14]. Therefore, it is used in a wide range of concentration into pharmaceutical, cosmetic, and food products, with several applications. Despite the wide use, VE was still not explored in organogel systems. The popularization of organogels to replace trans-fat contents in foods [15] and the benefits over the sensory and transdermal delivery of molecules [16] drive the necessity to understand the effect that additives have over the gel formation. The variation of organogels' structures may be very detrimental to the formulation development.

In this research work, the influences of mixtures between vegetable oil (VO) and VE on the microstructures and rheological properties of CW and 12HSA organogels were verified. The VE at several concentrations in CW and 12HSA organogels were investigated using polarized microscopy, rheology, and SAXS, providing detailed structural and mechanical information.

\section{Results and Discussion}

The macroscopic evaluation showed rigid and transparent gels with little syneresis for 12HSA organogels, while soft and opaque organogels were observed for CW samples (Figure S1). The polarized microscopy observation (Figure 1) explained those findings, since a heterogeneous and discontinuous crystal distribution was observed for $\mathrm{CW}$ organogels (Figure 1A), instead of dense and homogeneous crystals in 12HSA samples (Figure 1B). Similar patterns were observed previously in other organogels with the same concentrations of those organogelators $[4,5]$. However, for CW organogels, a tighter pattern with more evenly distributed crystals may be seen since the natural origin and the variable composition of the CW [17]. 

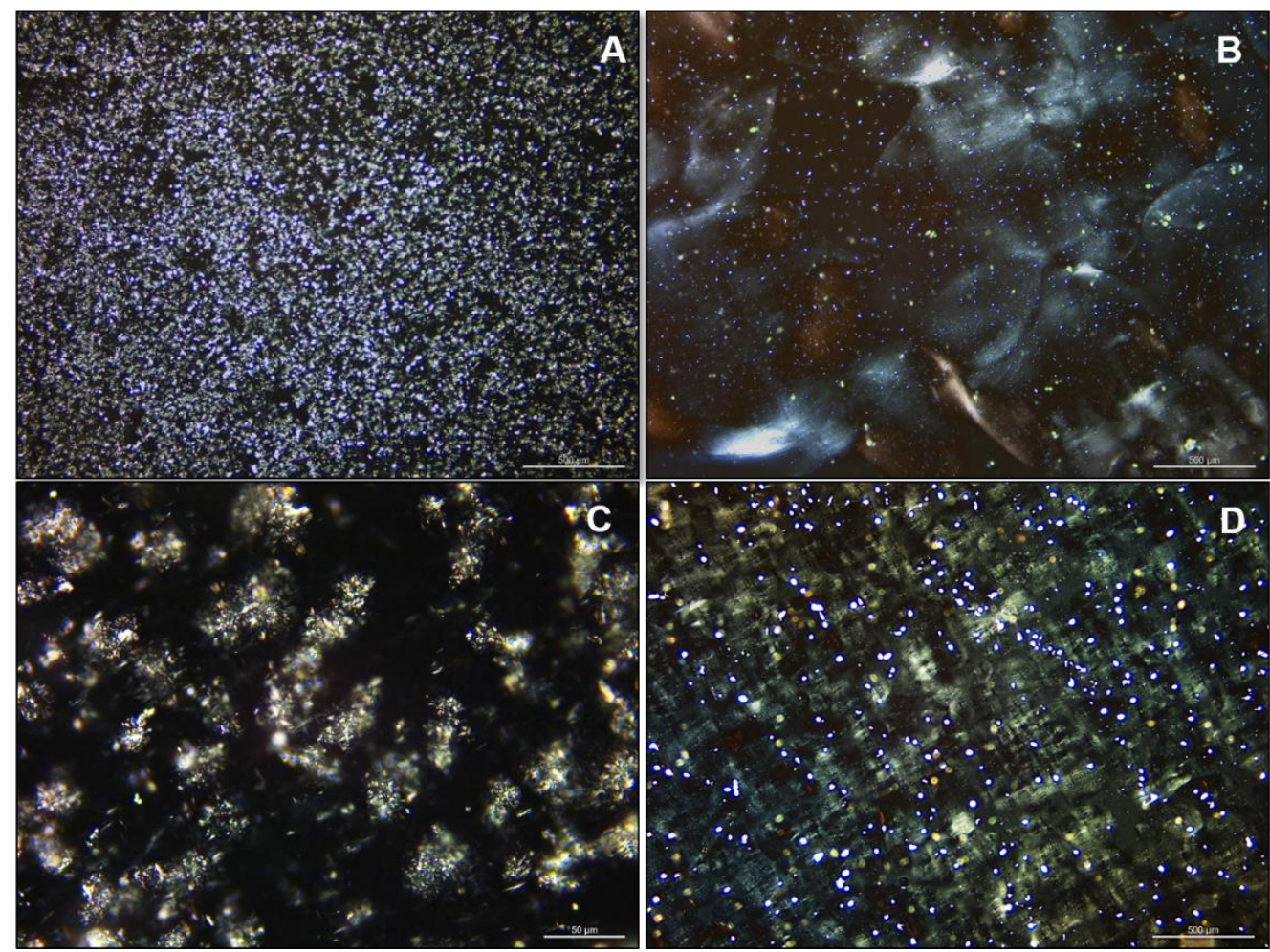

Figure 1. Polarized photomicroscopy images of organogels: (A) candelilla wax 2 (CW2):vegetable oil 97 (VO97):vitamin E 1 (VE1); (B) 12-hydroxystearic acid 2 (12HSA2):VO96:VE2; (C) CW2:VO98; and (D) 12HSA2:VO78:VE20. The scale bars are $500 \mu \mathrm{m}$ in (A,B,D), and the scale bar is $50 \mu \mathrm{m}$ in (C).

Throughout the concentration range of VE tested (0-20\%), the size and distribution of crystals were the same under polarized microscopy. The methodology was not a statistical image analysis; therefore, the rheology and SAXS investigations were used for better understanding the crystallization pattern of the organogels.

For all samples, in the frequency sweep measurements, the elastic moduli $\left(\mathrm{G}^{\prime}\right)$ dominated the viscous moduli $\left(G^{\prime \prime}\right)$, i.e., $G^{\prime}>G^{\prime \prime}$, throughout the range where the applied shear was evaluated (Figure S2), which marked solid-like behaviors of the gels [18]. We investigated the contribution of the VE concentration to the complex modulus $\left(\mathrm{G}^{*}\right)-\mathrm{a}$ combination of the $G^{\prime}$, the $G^{\prime \prime}$, and the phase angle-at $1 \mathrm{~Hz}$ for a comparison [19]. For the CW organogels, the $\mathrm{G}^{*}$ increased exponentially with the increasing of the VE content, while the exact opposite effect was observed for the 12HSA organogels. Apparently, the composition of the oily phase (VE and VO) affected the organization of the organogels, increasing the $\mathrm{G}^{*}$ values for $\mathrm{CW}$ and decreasing the $\mathrm{G}^{*}$ values for $12 \mathrm{HSA}$ (Figure 2 ). Higher $\mathrm{G}^{*}$ values were related with higher network gel strengths [20]. The phase angle was lower than the unity in the whole range of frequency investigated (Figure 2). Those results contributed to verifying the solid-like behaviors of all organogels [17]. The loss tangent was the tangent of the phase angle denoted as: $\tan \delta=\mathrm{G}^{\prime \prime} / \mathrm{G}^{\prime}$. The organogels containing $12 \mathrm{HSA}$ showed values of the loss tangent higher than 0.1 ( $\tan \delta>0.1)$, typical of the so-called weak gels, throughout the whole range of frequency tested [21]. However, the CW organogels presented an elastic behavior $(\tan \delta<0.1)$ at lower frequencies $(<1 \mathrm{~Hz})$ from 0 to $5 \% \mathrm{VE}$ concentrations [22]. When higher VE amounts were used $(20 \%)$, the behavior changed to weak gels $(\tan \delta>0.1)$. The VE hydrophilic area can impact somehow the arrangement of crystals, as described earlier, in combination with lecithin organogels [23]. In addition, when increasing the VE concentration, we proportionally reduced the amount of VO, which impacted the oily phase composition and could have changed its physical properties for crystal binding. 


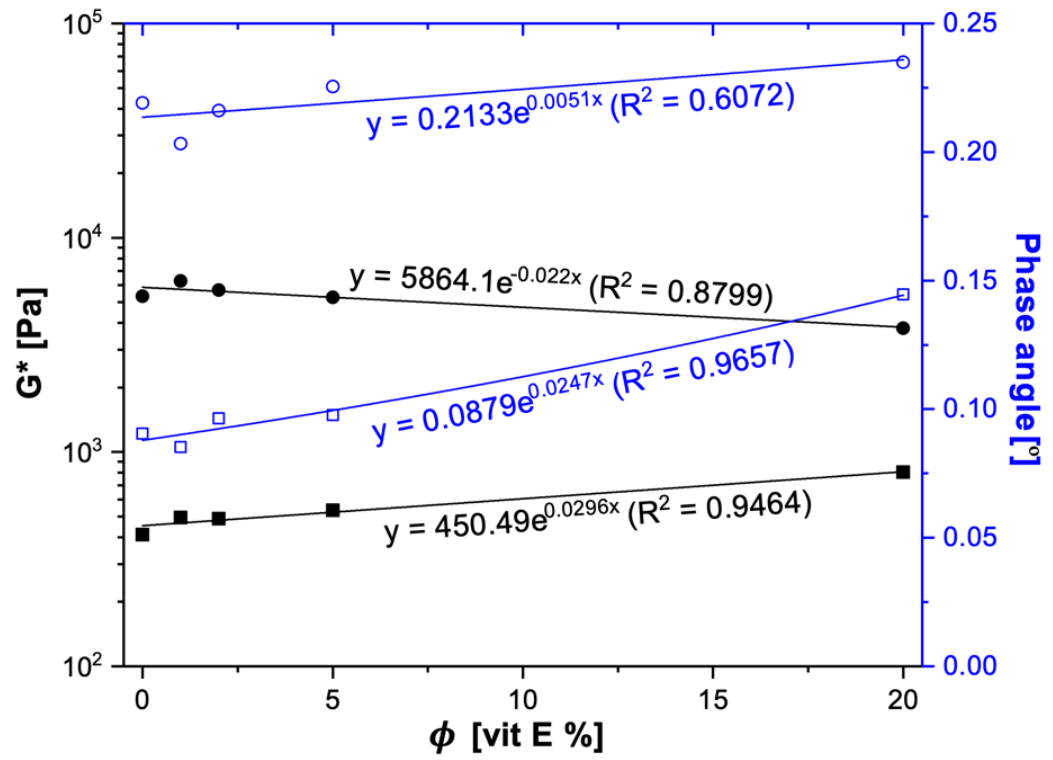

Figure 2. Frequency sweep measurements results for formulations with $12 \mathrm{HSA}: \mathrm{VO}(\bullet)$ and $\mathrm{CW}: \mathrm{VO}$ $(\boldsymbol{\square})$ in terms of $\mathrm{G}^{*}$ (closed symbols) and the phase angle (open symbols) at $1 \mathrm{~Hz}$. The experimental data are represented by symbols, and the fitting model is represented by lines according to the percentage of VE available $(\Phi)$.

The flow curve measurements showed a non-Newtonian shear-thinning behavior for all samples, characterized by a decrease in the viscosity as the shear rate increased (Figure 3A,B). The values of the parameters obtained using the Carreau-Yasuda model (Equation (13)) are shown in Table 1 and confirmed the shear-thinning behavior $(\eta<1)$. Moreover, because of the smaller relaxation time $(\lambda)$ values, no restriction of mobility was observed for the crystal network inside the organogels [24]. Closer crystals showed slower relaxation times, as observed for the $\mathrm{CW}$ organogels. The shear-thinning property is particularly interesting for applications that require the material to flow under an applied force, such as implants [25], topical products [26], injectable products [27], and foods [28]. The viscosity was higher for 12HSA organogels than that of the CW ones, which corroborated the microscopy findings earlier described. A more densely packed crystal network, such as seen for 12HSA organogels, can reduce the mobility of the components inside the organogels by increasing the tortuosity of the system [29]. Crystals acted as barriers against the organogel flow during the viscosity analysis.

The zero-shear viscosity $\left(\eta_{0}\right)$ is an important parameter to evaluate the consistency (the initial viscosity when the sample starts to flow). Consistency can be is translated as a physical attribute of the first contact between skin and formulation for topical products [30], stability [31], and package design [32]. For all compositions, an initial plateau was observed followed by a large drop in viscosity (shear-thinning region) and finally a second plateau (infinite shear viscosity). This behavior is characteristic of shear-thinning fluids, and it was more accentuated for 12HSA organogels (Figure 3B). The composition of the oily phase affected the consistency of the samples. When higher amounts of VE (and proportionally lower amounts of $\mathrm{VO}$ ) were used, we observed an increase in the consistency for CW organogels and a decrease in the consistency for 12HSA organogels. However, the 12HSA organogels showed naturally much higher $\eta_{0}$ values. Along with the $\mathrm{G}^{*}$ results, the consistency suggested that the composition of the oily phase affected crystal organization into the organogels. Earlier studies showed that the 12HSA network can be disrupted when lecithin is used in association as a co-oleogelator [33]. Likewise, lecithin showed a synergic effect with candelilla and other natural waxes on oleogels formation [15]. In our case, VE played a role as a co-organogelator, along with the reduction of the $\mathrm{VO}$ availability. Less oil could hinder the fibrous network for 12HSA, while alleviating the necessity of oil support 
for the CW network. Another hypothesis is that the combination of VE and VO presents singular physical-chemical properties for organogelation.
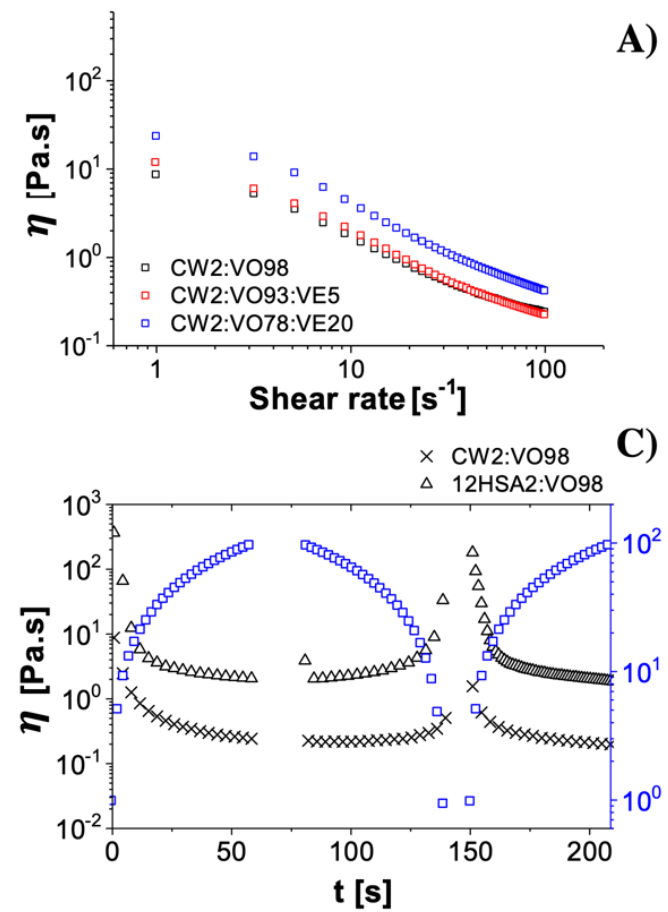

A)

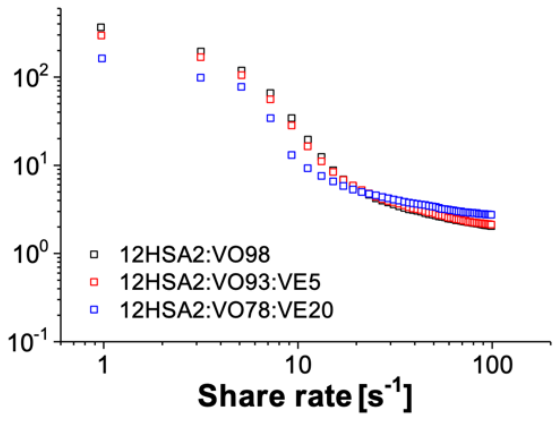

$\times \quad$ CW2:VO78:VE20

$\triangle$ 12HSA2:VO78:VE20

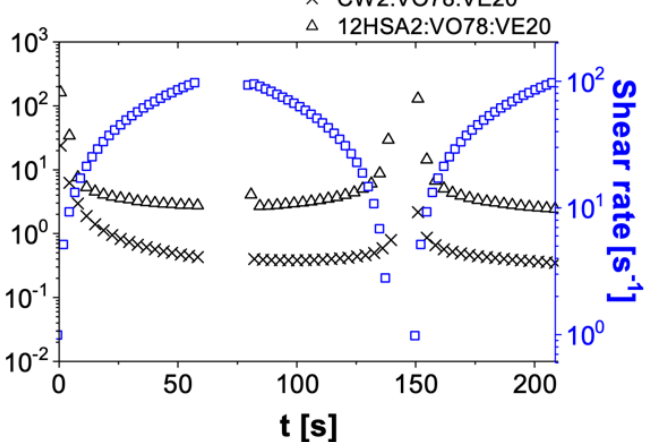

Figure 3. Viscosity $(\eta)$ versus shear rate for CW organogels (A) and for 12HSA organogels (B). Structure recovery after shear stress (black dots) for the organogels without VE (C) and with VE (D).

Table 1. Fitting parameters of the Carreau-Yasuda equation for flow curves and approximate phase transition temperatures Tmelt (melting temperature) and Tgel (gelation temperature).

\begin{tabular}{cccccccc}
\hline Code & $\begin{array}{c}\eta_{0} \\
(\mathbf{P a} \cdot \mathbf{s})\end{array}$ & $\begin{array}{c}\eta_{\infty} \\
\mathbf{( P a} \cdot \mathbf{s})\end{array}$ & $\lambda$ (s) & $\boldsymbol{n}$ & $\mathbf{a}$ & Tmelt $\left({ }^{\circ} \mathbf{C}\right)$ & Tgel $\left({ }^{\circ} \mathbf{C}\right)$ \\
\hline CW2:VO98 & $9.37 \pm 0.03$ & $0.15 \pm 0.01$ & $0.39 \pm 0.01$ & $-0.26 \pm 0.01$ & $2.15 \pm 0.03$ & 55 & 34 \\
CW2:VO93:VE5 & $16.06 \pm 1.26$ & $0.14 \pm 0.01$ & $0.38 \pm 0.02$ & $-0.44 \pm 0.33$ & $1.24 \pm 0.03$ & 55 & 38 \\
CW2:VO78:VE20 & $24.93 \pm 0.18$ & $0.22 \pm 0.01$ & $0.41 \pm 0.01$ & $-0.29 \pm 0.02$ & $2.49 \pm 0.04$ & 50 & 39 \\
12HSA2:VO98 & $350.08 \pm 8.80$ & $2.19 \pm 0.01$ & $0.27 \pm 0.01$ & $-1.65 \pm 0.01$ & $3.01 \pm 0.01$ & 72 & 66 \\
12HSA2:VO93:VE5 & $284.41 \pm 7.25$ & $2.21 \pm 0.01$ & $0.29 \pm 0.01$ & $-1.40 \pm 0.01$ & $3.63 \pm 0.01$ & 63 & 58 \\
12HSA2:VO78:VE20 & $150.89 \pm 4.09$ & $2.94 \pm 0.01$ & $0.29 \pm 0.01$ & $-1.39 \pm 0.01$ & $4.82 \pm 0.01$ & 59 & 46 \\
\hline
\end{tabular}

The time-dependency behavior and the structure recovery after stress are the two phenomenon involved with thixotropic fluids [34]. Thixotropy plays an important role in the sensory and stability features of topical and oral formulations. It is related with the spreadability of topical formulations over skin [35] and to predict the deformation of foods during the processing and handling operations [36]. The flow curves showed a recovery in the 12HSA viscosity after shearing at the same extend, despite the alterations of the oily phase. However, no recovery was observed for the CW organogels (Figure 3C,D). When a shear force is applied over oleogels, the ability to retain oil decreases since small crystals are formed [29]. In our experiment, the shear applied over CW organogels, which naturally showed a small crystals distribution, probably promoted an oil migration and increased the difficulty in recovering the original structure.

The thermal behavior of the gel-sol or sol-gel phase transition domains (PTDs) of organogels was evaluated using a temperature ramp test [37]. All PTDs shifted to smaller temperatures proportionally to the addition of VE (Figures 4 and 5). However, the shift was more accentuated for the 12HSA organogels. The melting temperature (Tmelt) for the 
12HSA organogels without VE was close to $79^{\circ} \mathrm{C}$, as reported by Esposito and coworkers [38] at the same concentration, but higher than $37^{\circ} \mathrm{C}$ for the gelation temperature (Tgel). Besides the different rheometer geometries and oily phases, the authors used strategies that could impact the crystallization pattern, such as a slower cooling rate compared to in our experiment $\left(1^{\circ} \mathrm{C} / \mathrm{min}\right)$, a surfactant, and a co-solvent $[3,37]$. They pointed that the addition of the surfactant disrupts the crystalline network, causing the reduction of the gel density, which was similar with our findings for VE. VE also impacts more complex systems, such as nanoemulsions. A decrease in the temperature of fat crystallization in oil-in-water stearin-rich milk fractions was observed for sodium caseinate-stabilized nanoemulsion containing VE [39]. Several works have studied the impact of VE in cellular membrane models using phospholipids. Alpha tocopherol, specifically, works in bilayer membranes' de-stabilization by forming complexes with lipid components [40]. This mechanism is plausible to explain the reduction of PTD temperatures in our results; nevertheless, the specific interaction between VE and the organogelators is currently unknown. In this sense, another hypothesis is that VE modifies the oily phase properties, consequently impacting the rheological properties of the organogel itself.
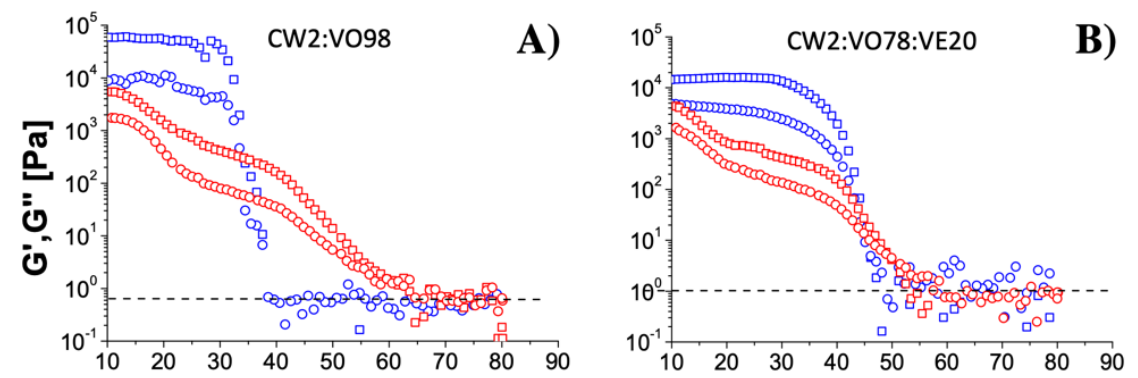

$\square$ Elastic moduli (G')

O Viscous moduli (G”)
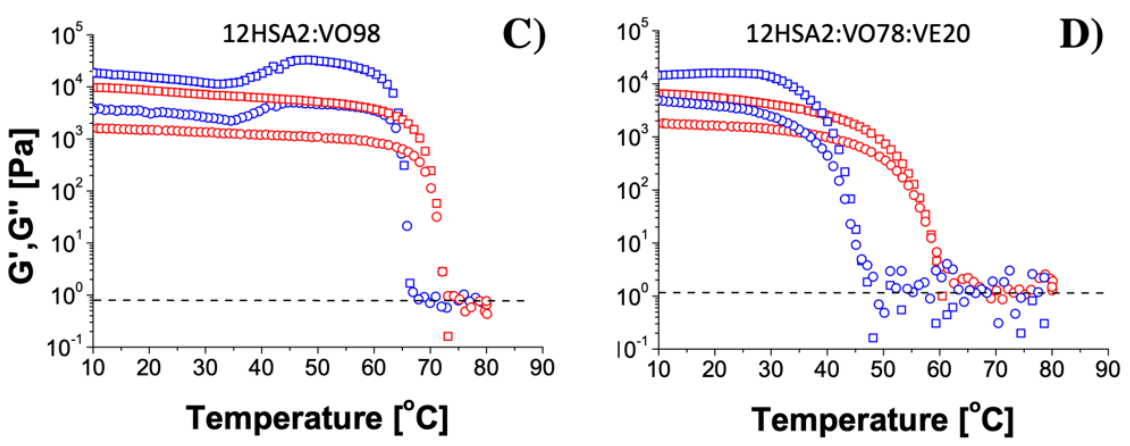

Cooling

Figure 4. Temperature ramp test results for CW2:VO98 (A), CW2:VO78:VE20 (B), 12HSA2:VO98 (C), and 12HSA2:VO78:VE20 (D) organogels. In Table 1, the organogel melting temperature and the gelation transition temperature obtained from the ramps where $\mathrm{G}^{\prime} \approx \mathrm{G}^{\prime \prime}$ are presented.

For the CW organogels, the pattern of the heating curve showed a gradual melting process, different from the sharp decrease in $\mathrm{G}^{\prime}$ for 12HSA. CW is a mixture of n-alkanes, esters of acids, alcohols, sterols, and free acids [9]. Each component has its own melting point and impacts the total organogel Tmelt, as well as in the interactions with VE, if they exist. Interestingly, the cooling ramp seemed to organize the crystals, since the cooling rate was controlled $\left(5^{\circ} \mathrm{C} / \mathrm{min}\right)$, opposing to the organogel production when it was naturally cooled. This may explain the higher $\mathrm{G}^{\prime}$ values after the temperature ramp test. Regarding this aspect, the 12HSA samples recovered approximatively their initial G' values (Figure 4C,D), while the $\mathrm{CW}$ organogels increased 10 times the initial $\mathrm{G}^{\prime}$ magnitude (Figure 4A,B), reassuring a thixotropic effect for the 12HSA organogels but not for the CW organogels. No significant changes were observed when changing the oily phase, except for the discrete reduction of PTD temperatures previously described. 


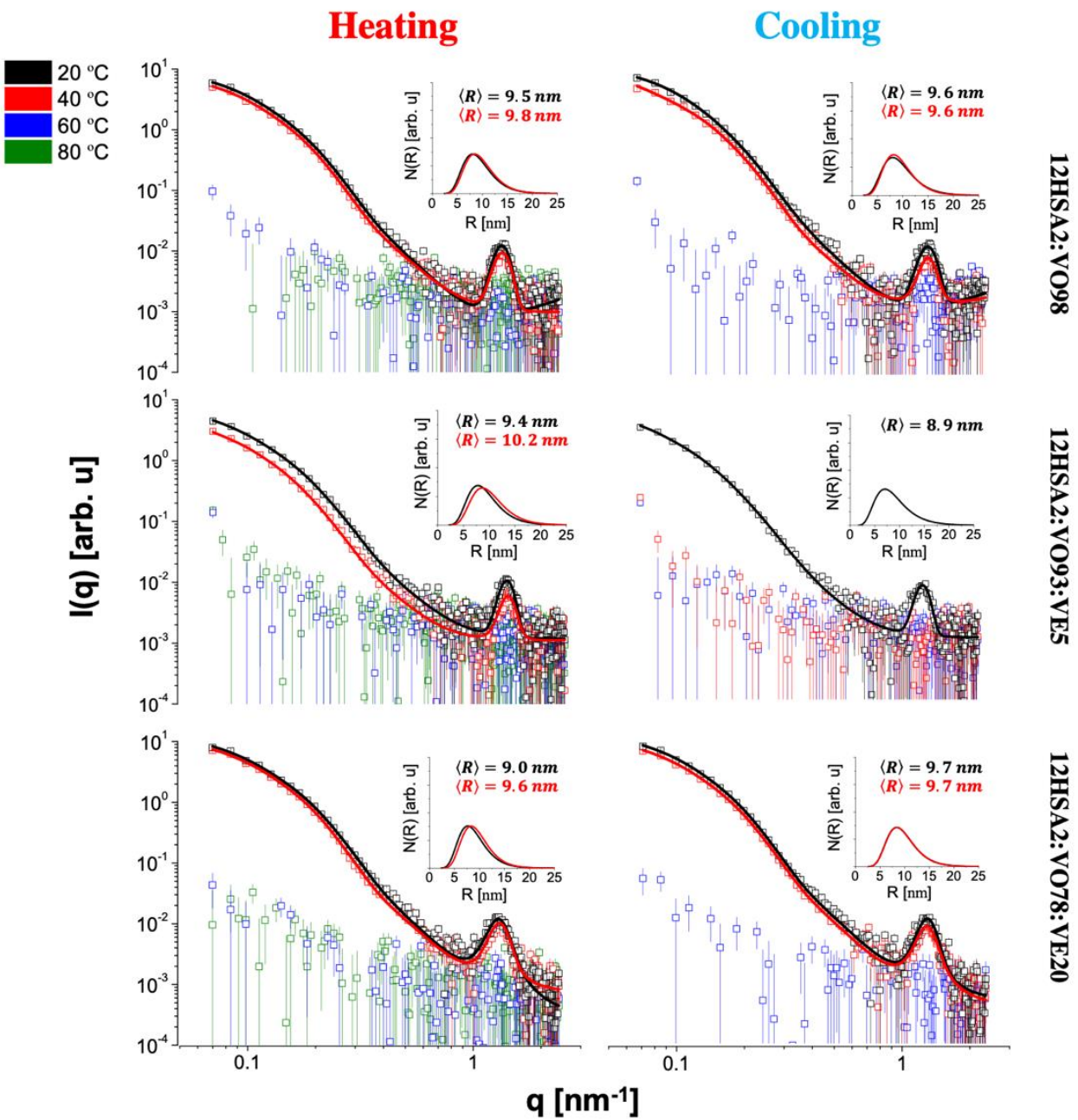

Figure 5. Small-angle X-ray scattering (SAXS) data for the 12-HSA organogels on heating and on cooling (open symbols) and fitted with Equation (12) (continuous lines). The insets correspond to the cylinder radius size distributions. The average radius for each distribution is shown as well.

To better understand the effect of the oily phase over the network structure at a nanoscale, SAXS experiments were performed, which brought important information about the shape and size of the gel nanostructure [41]. The SAXS data for the 12HSA and $\mathrm{CW}$ organogels (open symbols) at all investigated temperatures for heating and on cooling are shown in Figures 5 and 6, respectively. In the studied length scale, the profiles of the curves obtained for all organogelators were different, suggesting nanostructures with a distinct size and/or shape. A peak at $q$ of approximately $1.3 \mathrm{~nm}^{-1}$ was observed for the 12HSA organogels and was attributed to the (001) Bragg reflection of the 12HSA crystal [42]. It is interesting to note that even $\mathrm{CW}$ also has a crystalline structure according to the $\mathrm{X}$-ray diffraction data from previous works [43,44], which was not observed in our data. Possibly, its neatly ordered molecular arrangement was disrupted in the organogel formation, since its main component hentriacontane $(\sim 79 \%, w / w)$ is highly soluble in organic solvents, such as VOs [5]. 
Heating

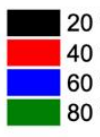
$20^{\circ} \mathrm{C}$
$40^{\circ} \mathrm{C}$
$60^{\circ} \mathrm{C}$
$80^{\circ} \mathrm{C}$

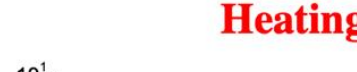

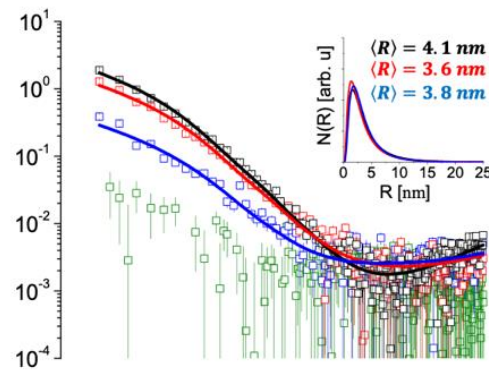
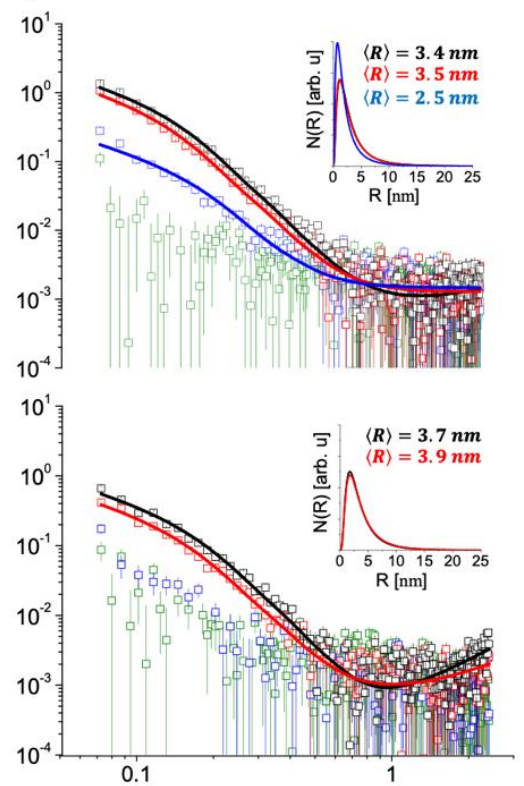

Cooling
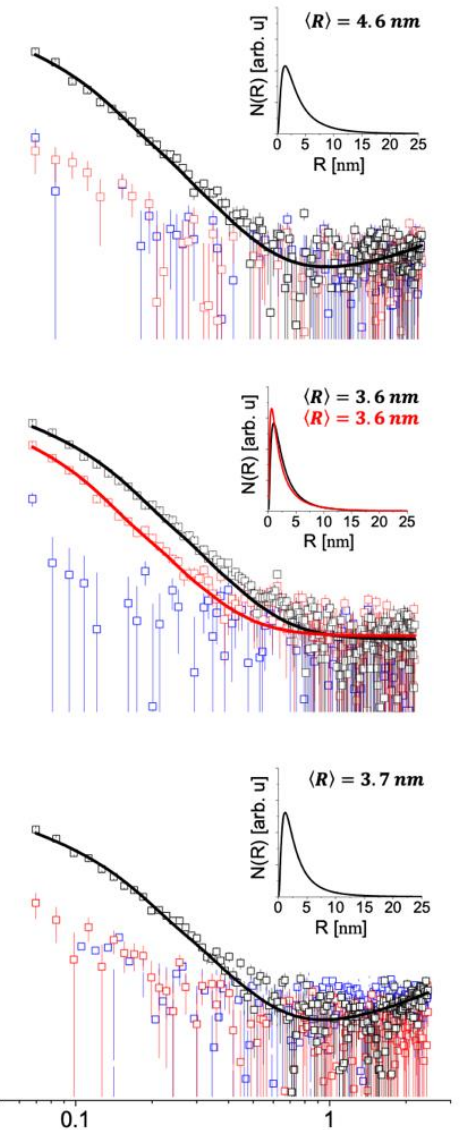

莡

Figure 6. SAXS data for the CW organogels on heating and on cooling (open symbols) and fitted with Equation (12) (continuous lines). The insets correspond to the cylinder radius size distributions. The average radius for each distribution is shown as well.

On heating, a structural gel-to-sol transition was clearly seen for all materials. After this event, the sample scattering curve practically coincided with the sunflower oil curve (Figure 7), the major component used in the sample preparation, and the data reduction led to very noisy curves. This fact made it easier to identify, in Figures 5 and 6, the mentioned transition, which occurred at $T \geq 40^{\circ} \mathrm{C}$ for all 12HSA compositions and CW2:VO78:VE20 and only at $T \geq 60^{\circ} \mathrm{C}$ for all CW compositions, except CW2:VO78:VE20, in agreement with the temperature ramp tests. Furthermore, the $\mathrm{CW}$ organogels seemed to be more thermalresistant than the ones composed of 12HSA, in the sense that they kept their nanostructures at high temperatures up to, at least, $60^{\circ} \mathrm{C}$, except CW2:VO78:VE20. For this particular composition, it was unclear if the melting of the gel at lower temperatures (relative to the other compositions) was caused by a specific effect of VE over the organogel structure or if it was a direct result of change in the oily phase properties due to the addition of VE followed by the reduction of VO. Nevertheless, from all these observations, one can argue that the sol-gel transition is composition-dependent. 

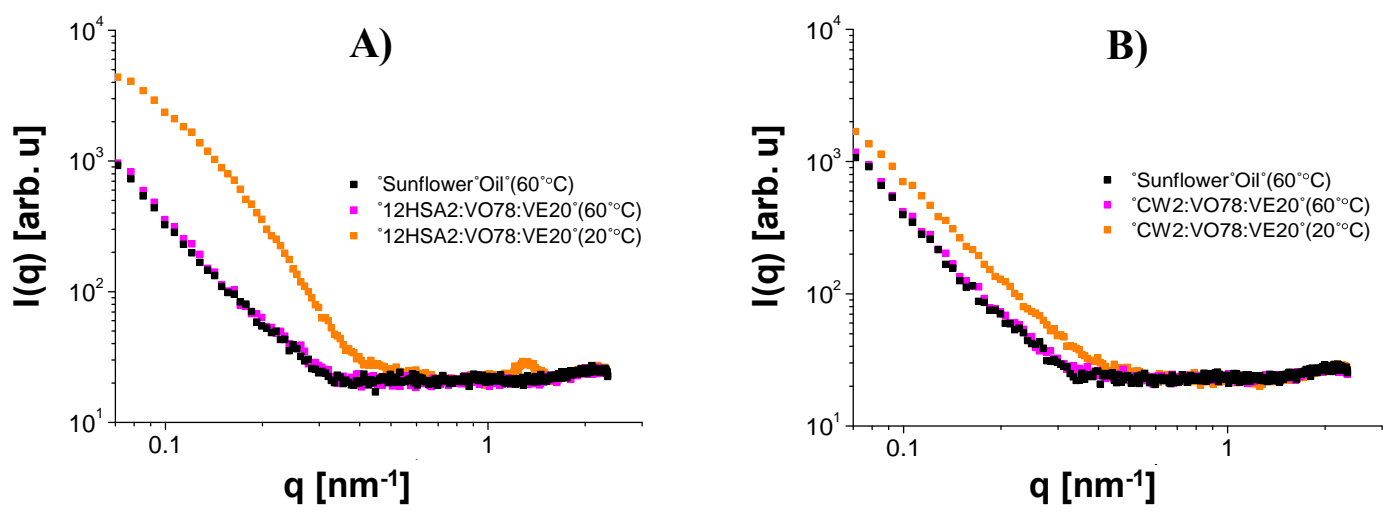

Figure 7. Comparison between the SAXS curves of sunflower oil at $60{ }^{\circ} \mathrm{C}$, the major component of the organogels presented in this manuscript, and the samples containing 12HSA (A) and CW (B), at 60 and $20^{\circ} \mathrm{C}$. In this example, the curves collected at $60^{\circ} \mathrm{C}$ are almost perfectly overlapped. This fact makes some of the treated curves presented in Figures 5 and 6 very noisy in all q values. For the other curves, like the ones collected at $20^{\circ} \mathrm{C}$, only their high q regions are noisy.

On cooling, the sol-to-gel transition was, in general, distinct from the one observed on heating, except for 12HSA2:VO98 and 12HSA2:VO78:VE20. Gels were formed at $T \leq 60{ }^{\circ} \mathrm{C}$ for all 12HSA compositions (except 12HSA2:VO93:VE5) and only at $T \leq 20{ }^{\circ} \mathrm{C}$ for all CW organogels (except CW2:VO93:VE5). The combination 93\% VO + 5\% VE seemed odd in both types of materials: with 12HSA, the gel formation was difficult, whereas with CW the exact opposite occurred. This fact corroborated once more that the particular composition of the gel had a great influence on its properties, including the sol-to-gel transition temperature. It is quite interesting to observe that the materials containing $\mathrm{CW}$ were less prompt to form gels on cooling, whereas the same gels on heating lasted longer at more high temperatures than the ones composed of 12HSA, as discussed before.

Aiming to retrieve quantitative information on the organogel nanostructure, the SAXS data were satisfactorily fitted using Equation (13) (continuous lines of Figures 5 and 6). From the fittings, the average radius, $\langle R\rangle$, and the size distributions of $R$ values (graphs insets in Figures 5 and 6) were obtained. For the 12HSA organogels, $\langle R\rangle$ was approximately $10 \mathrm{~nm}$, in agreement with the value reported for 12HSA gel in toluene [45], whereas for the CW materials, $\langle R\rangle$ was approximately $4 \mathrm{~nm}$. This difference in $\langle R\rangle$ might be directly associated to the differences observed in the polarized microscopy results. With the temperature increase, $\langle R\rangle$ slightly increased for all compositions containing $12 \mathrm{HSA}$, whereas there was no apparent pattern for the $\mathrm{CW}$ organogels. In general, $\langle R\rangle$ values on cooling were different compared to the ones obtained on heating. This sort of hysteresis was also observed in the measured rheological properties. Regarding the size distributions, they were positively skewed, i.e., the distributions had more data on the right tail. A dispersion of $R$ values around $30 \%$ was found in all cases, which was 1.5 times higher than the value found for 12HSA gel in toluene [45], likely related to the presence of sunflower oil. To the best of our knowledge, there is no reference value for CW organogels, and this is the first time that this kind of SAXS analysis is applied to this material. All in all, the SAXS data for both types of organogel pointed to the structural differences between them, which were satisfactorily quantified by the use of an advanced analysis. Moreover, the gel-to-sol transition on heating and the sol-to-gel transition on cooling were quite evident in the presented curves and strongly dependent on the composition of each investigated organogel.

\section{SAXS Model}

SAXS data were modeled assuming non-interacting randomly oriented cylindrical particles with radius $R$ and length $L$. In this case, the theoretical scattering intensity is given by:

$$
I(q)=s c \cdot P(q, R, L)+b a c k,
$$


where the parameter $s c$ is a scale factor and $P(q, R, L)$ is the cylinder normalized form factor [46]:

$$
P(q, R, L)=\int_{0}^{\frac{\pi}{2}}\left[\frac{2 J_{1}(q R \sin \alpha)}{q R \sin \alpha} \frac{\sin (0.5 q L \cos \alpha)}{0.5 q L \cos \alpha}\right]^{2} \sin \alpha d \alpha,
$$

where $J_{1}(x)$ is the first-order Bessel function and $\alpha$ is the angle between the axis of the cylinder and the scattering vector $\vec{q}$. In most of the samples was observed a non-flat curve at high $q$ values, related to a gel-buffer mismatch in data reduction. To compensate this effect, a non-linear background, back, was introduced. In our case, a simple second-order polynomial function satisfactorily fit this region of the SAXS curves.

Under the hypothesis, the cylinders are sufficiently long $(L \gg R)$, used in previous works [45]. Equation (2) can be approximated as a product of the longitudinal factor, $P_{\text {rod }}(q, L)$, parallel to the cylinder axis, and the scattering cross-section function, $P_{C S}(q, R)[47]$ :

$$
P(q, R, L)=P_{\text {rod }}(q, L) P_{C S}(q, R),
$$

where

$$
\begin{gathered}
P_{\text {rod }}(q, L)=\frac{\operatorname{Si}(q \cdot L)}{0.5 \cdot q L}-\left[\frac{\sin (0.5 \cdot q \cdot L)}{0.5 \cdot q \cdot L}\right]^{2}, \\
S i(x)=\int_{0}^{x} \frac{\sin t}{t} d t, \\
P_{C S}(q, R)=\left[A_{C S}(q, R)\right]^{2}, \\
A_{C S}(q, R)=\frac{2 \cdot J_{1}(q \cdot R)}{q \cdot R} .
\end{gathered}
$$

In this study, after several tests, $L$ was fixed at $200 \mathrm{~nm}$, which provided the best fittings and fulfilled the above hypothesis regarding long cylinders. Taking the $R$ polydispersity into account, the normalized form factor was written as [48]:

$$
\langle P(q, R, L)\rangle=\frac{\int_{-\infty}^{\infty} N(R) V(R, L)^{2} P(q, R, L) d R}{\int_{-\infty}^{\infty} N(R) V(R, L)^{2} d R},
$$

where $P(q, R, L)$ is defined by Equation (2) or Equation (3) and $V(R, L)=\pi R^{2} L$ is the cylinder volume. In this study, $N(R)$ was a log-normal distribution [49,50]:

$$
N(R)=\frac{1}{R \omega \sqrt{2 \pi}} \exp \left(-\frac{1}{2}\left(\frac{\ln R-\theta}{\omega}\right)^{2}\right),
$$

where $\theta$ and $\omega$ are the average and the standard deviation, respectively, of a Gauss distribution of $\ln R$. The expected (average) value for the variable $R$ and its standard deviation were described as $[49,50]$ :

$$
\begin{gathered}
\mu=\exp \left(\theta+\frac{\omega^{2}}{2}\right), \\
\sigma=\sqrt{\exp \left(2 \theta+\omega^{2}\right) \cdot\left(\exp \left(\omega^{2}\right)-1\right) .}
\end{gathered}
$$

Therefore, rewriting Equation (1), the final fitting equation was expressed as:

$$
I(q)=s c \cdot\langle P(q, R, L)\rangle+\text { back. }
$$

For the organogels containing 12HSA, its crystal structure contributed with a peak at $q$ of approximately $1.3 \mathrm{~nm}^{-1}$ (see Figure 5). This feature was taken into account by introducing a Gauss function term in Equation (1), as performed in previous works [45]. 


\section{Conclusions}

The design of the organogel's composition plays an important role in the physicalchemical properties of materials. We found distinct structures for all organogels' compositions. While 12HSA built a highly packed network, CW showed sparse crystals observed with polarized microscopy. All organogels were characterized as weak gels with a shear-thinning behavior, but only 12HSA organogels showed thixotropy. The gradual replacement of VO by VE in the oily phase showed an improvement of the gel strength for $\mathrm{CW}$, but a reduction for 12HSA organogels. Likewise, all phase transition temperatures were reduced in a dose-dependent pattern, especially for 12HSA. Larger crystals that slightly increased upon heating were observed via SAXS for 12HSA when compared with for CW. Phase transition was strongly dependent on the composition of the organogel. Further investigation regarding the deeper structure of organogels could clarify whether VO and VE form a new oily phase with different properties or if VE and organogelators compete for VO availability. Those data would be especially beneficial for pharmaceutical, cosmetics, and food exploitation of organogels.

\section{Materials and Methods}

\subsection{Organogel Preparation}

Sunflower (Helianthus annuus) oil (VO) with a high oleic content, was purchased from Agri Pure 80, Cargill Agrícola S/A, São Paulo, SP, Brazil. The organogelators CW and 12HSA were purchased from Double Refined Candelilla Wax 102P, Koster Keunen Inc, Wartertown, CT, USA, and A. Azevedo, São Paulo, SP, Brazil, respectively. VE (dl$\alpha$-Tocopherol) was purchased from DSM, Parsippany, NJ, USA. All materials were used as received. The production of organogels consisted in the mixing of VO with $\mathrm{CW}$ or $12 \mathrm{HSA}$ at $85^{\circ} \mathrm{C}$ upon continuously stirring at $200 \mathrm{rpm}$ (RW 20; IKA-Werke, Staufen, Germany), followed by the addition of VE once the organogelator was completely melted. The component amounts of each sample are shown in Table 2. At this point, the mixing was continued for $5 \mathrm{~min}$, followed by rest and cooling at room temperature for $24 \mathrm{~h}$. The batch sizes were standardized at $50 \mathrm{~g}$ to insure the same thermal heating transfer characteristics.

Table 2. Organogels studied in this work. The acronyms "CW", "VO", "12HSA", and "VE" mean candelilla wax, vegetable (sunflower) oil, 12-hydroxystearic acid, and vitamin E, respectively.

\begin{tabular}{cccccc}
\hline Code & $\% \mathrm{CW}(w / w)$ & $\%$ 12HSA $(w / w)$ & $\%$ VO $(w / w)$ & $\%$ MO $(w / w)$ & $\%$ VE $(w / w)$ \\
\hline CW2:VO98 & 2.0 & - & 98.0 & - & - \\
CW2:VO97:VE1 & 2.0 & - & 97.0 & - & 1.0 \\
CW2:VO96:VE2 & 2.0 & - & 96.0 & - & 2.0 \\
CW2:VO93:VE5 & 2.0 & - & 93.0 & - & 5.0 \\
CW2:VO78:VE20 & 2.0 & - & 98.0 & - & - \\
12HSA2:VO98 & - & 2.0 & 97.0 & - & 1.0 \\
12HSA2:VO97:VE1 & - & 2.0 & 96.0 & - & 2.0 \\
12HSA2:VO96:VE2 & - & 2.0 & 93.0 & - & 5.0 \\
12HSA2:VO93:VE5 & - & 2.0 & 78.0 & - & 20.0 \\
12HSA2:VO78:VE20 & - & 2.0 & &
\end{tabular}

\subsection{Microscopy Tests}

Polarized microscopy was performed in a DM2700, Leica Microsystems, Wetzlar, Germany, with a $40 \times$ objective (Leica HI Plan $40 \times / 0.65$ POL). The images were captured with a digital camera Leica MC120 HD (Leica Microsystems, Wetzlar, Germany) and analyzed with the LEICA Application Suite Software (Leica, Wetzlar, Germany). All samples were visualized over glass plates without dilution.

\subsection{Rheological Characterization}

Rheological measurements were performed in a strain rheometer (TA Instruments, DHR-2, New Castle, DE, USA) with the crosshatched parallel plates with a $20 \mathrm{~mm}$ geometry (gap: $300.0 \pm 0.1 \mu \mathrm{m}$ ) coupled to a Peltier system for temperature control. Approximately 
$0.5 \mathrm{~g}$ of organogels was transferred to the geometry and left still for 3 min to the equilibrium temperature of $25^{\circ} \mathrm{C}$ before each measurement. We conducted 3 different experiments in order to evaluate the elastic modulus $\left(G^{\prime}\right)$ and the viscous modulus $\left(G^{\prime \prime}\right)$ behavior when submitted to stress: frequency sweep tests, flow curve tests, and temperature ramp tests. All measurements were conducted in triplicates. Frequency sweep tests were carried out in the range of $0.1-100 \mathrm{rad} / \mathrm{s}$ at $25^{\circ} \mathrm{C}$ in the viscoelastic linear region previous evaluated by the amplitude sweep test for each sample. We monitored the $G^{\prime}$, the $G^{\prime \prime}$, and the phase angle $\left({ }^{\delta}\right)$. Three flow curve tests were performed in controlled stress conditions: increasing the stress from 0 up to $100 \mathrm{~s}^{-1}$, decreasing from 100 to $0 \mathrm{~s}^{-1}$, and increasing again from 0 to $100 \mathrm{~s}^{-1}$. Intervals of $60 \mathrm{~s}$ were adopted between every curve at $25{ }^{\circ} \mathrm{C}$. We monitored the viscosity $(\eta)$ behavior during shear stress. The Carreau-Yasuda viscosity model [51] was used to fit the flow curves:

$$
\eta(\gamma)-\eta_{\infty}=\left(\eta_{0}-\eta_{\infty}\right)\left[1+(\lambda \gamma)^{a}\right]^{\frac{(n-1)}{a}}
$$

where $\eta$ is the shear-dependent viscosity, $\eta_{0}$ is the zero-shear viscosity, $\eta_{\infty}$ is the infinite shear viscosity, $\lambda$ is the relaxation time, $a$ is a parameter describing the rate of the transition from the Newtonian plateau to the power law region, and $\mathrm{n}$ is the power law index.

Dynamic temperature ramp measurements were performed at $1 \mathrm{~Hz}$ in the linear viscoelastic regime, ranging from 10 to $70{ }^{\circ} \mathrm{C}$ and from 70 to $10{ }^{\circ} \mathrm{C}$, with intervals of $60 \mathrm{~s}$ between curves in a ramp rate of $5^{\circ} \mathrm{C} / \mathrm{min}$.

\subsection{SAXS}

SAXS measurements were performed using a Nanostar (Bruker) instrument equipped with a microfocus Genix 3D system (Xenocs). The samples were maintained in quartz capillaries with a mean diameter of $1.5 \mathrm{~mm}$, and the scattered intensity was collected with a 2D Vantec-2000 detector. The sample-to-detector distance was $\sim 1 \mathrm{~m}$, which provided an effective range of the modulus of the transfer moment vector $q$ experimentally accessible of $0.08-2.3 \mathrm{~nm}^{-1}$, with $\mathrm{q}=4 \pi \sin (\theta) / \lambda \mathrm{s}$, where $2 \theta$ is the scattering angle and $\lambda \mathrm{s}=0.154 \mathrm{~nm}$ is the $\mathrm{X}$-ray wavelength from the copper $\mathrm{K} \alpha$ radiation). The sample temperature was varied using a circulating water bath, with a precision of $0.1^{\circ} \mathrm{C}$. An equilibration time of $600 \mathrm{~s}$ was applied, after the samples reached one of the selected temperatures $(20,40,60$, and $80^{\circ} \mathrm{C}$ ). For the treatment of the $1 \mathrm{D}$ data, obtained through the azimuthal integration of the 2D data, the SUPERSAXS package [52] was used and consisted of normalization by the measuring time (1800 s) and sample transmission, followed by the subtraction of the blank scattering. The scattering from the sunflower oil, measured at the same temperatures of the samples, was taken as the blank, since it is the major component of these organogels.

Supplementary Materials: The following are available online at https: / www.mdpi.com/article / 10.3390/gels8010036/s1, Figure S1: Macroscopic aspecst of organogels: (A) 12HSA2:VO98; (B) 12HSA2:VO78:VE20; (C) CW2:VO98; (D) CW2:VO78:VE20, Figure S2: Frequency sweep test results: (A) 12HSA2:VO96:VE2; (B) 12HSA2:VO78:VE20; (C) CW2:VO96:VE2; (D) CW2:VO78:VE20.

Author Contributions: R.M.M.: Conceptualization, methodology, formal analysis, investigation, writing-Original Draft, and writing - review and editing; P.L.O.F.: methodology, formal analysis, investigation, and writing — the original draft; B.B.G.: formal analysis, investigation, writing — the original draft; W.V.M.: supervision and funding acquisition; M.V.R.V.: supervision and funding acquisition; S.C.d.S.L.: supervision and funding acquisition; C.L.P.d.O.: writing-review and editing, supervision, and funding acquisition; C.R. and A.R.B.: writing-review and editing, supervision, and funding acquisition. All authors have read and agreed to the published version of the manuscript.

Funding: A.R.B. acknowledges Conselho Nacional de Desenvolvimento Científico e Tecnológico (CNPq; process 305250/2019-1) and São Paulo Research Foundation (FAPESP, process 2019/16169-0). R.M.M. acknowledges Coordenação de Aperfeiçoamento de Pessoal de Nível Superior-Brasil (CAPES) Finance Code 001. C.L.P.d.O. acknowledges FAPESP (Thematic Project; 2016/24531-3), INCT-FCx (Instituto Nacional de Ciência e Tecnologia de Fluidos Complexos) and CNPq Scholarship-Brazil (303001/2019-4). P.L.O.F. acknowledges FAPESP (processes 2019/12301-1 and 2020/13204-7). B.B.G 
acknowledges FAPESP (processes 2018/05888-3 and 2020/02192-8). C.R. acknowledges Foundation for Science and Technology (FCT) projects UIDB/04567/2020 and UIDP/04567/2020.

Institutional Review Board Statement: Not applicable.

Informed Consent Statement: Not applicable.

Conflicts of Interest: The authors declare no conflict of interest.

\section{References}

1. Esposito, C.L.; Kirilov, P.; Roullin, V.G. Organogels, promising drug delivery systems: An update of state-of-the-art and recent applications. J. Control. Release 2018, 271, 1-20. [CrossRef]

2. Rogers, M.A.; Marangoni, A.G. Acid in Vegetable Oils \& DESIGN 2008. Cryst. Growth Des. 2008, 8, $4596-4601$.

3. Toro-Vazquez, J.F.; Morales-Rueda, J.; Torres-Martínez, A.; Charó-Alonso, M.A.; Mallia, V.A.; Weiss, R.G. Cooling rate effects on the microstructure, solid content, and rheological properties of organogels of amides derived from stearic and (R)-12- hydroxystearic acid in vegetable oil. Langmuir 2013, 29, 7642-7654. [CrossRef] [PubMed]

4. Co, E.D.; Marangoni, A.G. Organogels: An alternative edible oil-structuring method. J. Am. Oil Chem. Soc. 2012, 89, 749-780. [CrossRef]

5. Toro-Vazquez, J.; Morales-Rueda, J.A.; Dibildox-Alvarado, E.; Charó-Alonso, M.; Alonzo-Macias, M.; González-Chávez, M. Thermal and textural properties of organogels developed by candelilla wax in safflower oil. J. Am. Oil Chem. Soc. 2007, 84, 989-1000. [CrossRef]

6. Morales-Rueda, J.A.; Dibildox-Alvarado, E.; Charó-Alonso, M.A.; Weiss, R.G.; Toro-Vazquez, J.F. Thermo-mechanical properties of candelilla wax and dotriacontane organogels in safflower oil. Eur. J. Lipid Sci. Technol. 2009, 111, 207-215. [CrossRef]

7. Rogers, M.A.; Wright, A.J.; Marangoni, A.G. Crystalline stability of self-assembled fibrillar networks of 12-hydroxystearic acid in edible oils. Food Res. Int. 2008, 41, 1026-1034. [CrossRef]

8. Dalkas, G.; Matheson, A.B.; Vass, H.; Gromov, A.; Lloyd, G.O.; Koutsos, V.; Clegg, P.S.; Euston, S.R. Molecular Interactions behind the Self-Assembly and Microstructure of Mixed Sterol Organogels. Langmuir 2018, 34, 8629-8638. [CrossRef]

9. Matheson, A.B.; Dalkas, G.; Gromov, A.; Euston, S.R.; Clegg, P.S. The development of phytosterol-lecithin mixed micelles and organogels. Food Funct. 2017, 8, 4547-4554. [CrossRef]

10. Martinez, R.M.; Magalhães, W.V.; Sufi, B.S.; Padovani, G.; Nazato, L.I.S.; Velasco, M.V.R.; da Silva Lannes, S.C.; Baby, A.R. Vitamin E-loaded bigels and emulsions: Physicochemical characterization and potential biological application. Colloids Surf. B Biointerfaces 2021, 201, 111651. [CrossRef]

11. Butt, H.; Mehmood, A.; Ali, M.; Tasneem, S.; Anjum, M.S.; Tarar, M.N.; Khan, S.N.; Riazuddin, S. Protective role of vitamin E preconditioning of human dermal fibroblasts against thermal stress in vitro. Life Sci. 2017, 184, 70. [CrossRef]

12. Delinasios, G.J.; Karbaschi, M.; Cooke, M.S.; Young, A.R. Vitamin E inhibits the UVAI induction of "light" and "dark" cyclobutane pyrimidine dimers, and oxidatively generated DNA damage, in keratinocytes. Sci. Rep. 2018, 8, 423. [CrossRef]

13. Shi, K.; Lio, P.A. Alternative Treatments for Atopic Dermatitis: An Update. Am. J. Clin. Dermatol. 2019, 20, 251-266. [CrossRef]

14. Mohanan, A.; Nickerson, M.T.; Ghosh, S. Oxidative stability of flaxseed oil: Effect of hydrophilic, hydrophobic and intermediate polarity antioxidants. Food Chem. 2018, 266, 524-533. [CrossRef]

15. Puscas, A.; Muresan, V.; Socaciu, C.; Muste, S. Oleogels in Food: A Review of Current and Potential Applications. Foods 2020, 9, 70. [CrossRef] [PubMed]

16. Alsaab, H.; Bonam, S.P.; Bahl, D.; Chowdhury, P.; Alexander, K.; Boddu, S.H.S. Organogels in drug delivery: A special emphasis on organogels pluronic lecithin. J. Pharm. Pharm. Sci. 2016, 19, 252-273. [CrossRef]

17. Rocha, J.C.B.; Lopes, J.D.; Mascarenhas, M.C.N.; Arellano, D.B.; Guerreiro, L.M.R.; da Cunha, R.L. Thermal and rheological properties of organogels formed by sugarcane or candelilla wax in soybean oil. Food Res. Int. 2013, 50, 318-323. [CrossRef]

18. Patel, A.R.; Babaahmadi, M.; Lesaffer, A.; Dewettinck, K. Rheological Profiling of Organogels Prepared at Critical Gelling Concentrations of Natural Waxes in a Triacylglycerol Solvent. J. Agric. Food Chem. 2015, 63, 4862-4869. [CrossRef] [PubMed]

19. Lupi, F.R.; Gentile, L.; Gabriele, D.; Mazzulla, S.; Baldino, N.; de Cindio, B. Olive oil and hyperthermal water bigels for cosmetic uses. J. Colloid Interface Sci. 2015, 459, 70-78. [CrossRef]

20. Luo, Y.; Liu, X.; Pang, Z. Tribo-rheological properties of acid milk gels with different types of gelatin: Effect of concentration. J. Dairy Sci. 2019, 102, 7849-7862. [CrossRef] [PubMed]

21. Ikeda, S.; Nishinari, K. "Weak gel"-type rheological properties of aqueous dispersions of nonaggregated K-carrageenan helices. J. Agric. Food Chem. 2001, 49, 4436-4441. [CrossRef] [PubMed]

22. Avanza, M.V.; Puppo, M.C.; Añón, M.C. Rheological characterization of amaranth protein gels. Food Hydrocoll. 2005, 19, 889-898. [CrossRef]

23. Nikiforidis, C.V.; Scholten, E. Self-assemblies of lecithin and $\alpha$-tocopherol as gelators of lipid material. RSC Adv. 2014, 4 , 2466-2473. [CrossRef]

24. Zare, Y.; Park, S.P.; Rhee, K.Y. Analysis of complex viscosity and shear thinning behavior in poly (lactic acid)/poly (ethylene oxide)/carbon nanotubes biosensor based on Carreau-Yasuda model. Results Phys. 2019, 13, 102245. [CrossRef] 
25. Ilyin, S.O.; Kulichikhin, V.G.; Malkin, A.Y. The rheological characterisation of typical injection implants based on hyaluronic acid for contour correction. Rheol. Acta 2016, 55, 223-233. [CrossRef]

26. Carriço, C.; Pinto, P.; Graça, A.; Gonçalves, L.M.; Ribeiro, H.M.; Marto, J. Design and characterization of a new quercus suber-based pickering emulsion for topical application. Pharmaceutics 2019, 11, 131. [CrossRef]

27. Pang, Y.; Liu, J.; Moussa, Z.L.; Collins, J.E.; McDonnell, S.; Hayward, A.M.; Jajoo, K.; Langer, R.; Traverso, G. Endoscopically Injectable Shear-Thinning Hydrogels Facilitating Polyp Removal. Adv. Sci. 2019, 6, 1901041. [CrossRef]

28. Espinosa, L.; To, N.; Symoneaux, R.; Renard, C.M.G.C.; Biau, N.; Cuvelier, G. Effect of processing on rheological, structural and sensory properties of apple puree. Procedia Food Sci. 2011, 1, 513-520. [CrossRef]

29. Valoppi, F.; Salmi, A.; Ratilainen, M.; Barba, L.; Puranen, T.; Tommiska, O.; Helander, P.; Heikkilä, J.; Haeggström, E. Controlling oleogel crystallization using ultrasonic standing waves. Sci. Rep. 2020, 10, 14448. [CrossRef]

30. Lupi, F.R.; Shakeel, A.; Greco, V.; Oliviero Rossi, C.; Baldino, N.; Gabriele, D. A rheological and microstructural characterisation of bigels for cosmetic and pharmaceutical uses. Mater. Sci. Eng. C 2016, 69, 358-365. [CrossRef]

31. Malvern Panalytical Multiple Ways to Optimize Rheology for Increased Dispersion, Colloidal and Emulsion Stability. Available online: https: / / www.azom.com/article.aspx?ArticleID=11442 (accessed on 10 August 2021).

32. Carrà, S.; Chiozza, F.; Curto, F.; Ferraioli, M. Rheology to understand operational parameter, as a predictive method to avoid off specifications and maximizing the packaging process. Annu. Trans. Nord. Rheol. Soc. 2015, 23, 135-142.

33. Okuro, P.K.; Martins, A.J.; Vicente, A.A.; Cunha, R.L. Perspective on oleogelator mixtures, structure design and behaviour towards digestibility of oleogels. Curr. Opin. Food Sci. 2020, 35, 27-35. [CrossRef]

34. Alghooneh, A.; Razavi, S.M.A.; Kasapis, S. A New Approach to Distinguish Thixotropic and Viscoelastic Phenomena. Food Biophys. 2020, 15, 72-84. [CrossRef]

35. Estanqueiro, M.; Amaral, M.H.; Sousa Lobo, J.M. Comparison between sensory and instrumental characterization of topical formulations: Impact of thickening agents. Int. J. Cosmet. Sci. 2016, 38, 389-398. [CrossRef] [PubMed]

36. Toker, O.S.; Karasu, S.; Yilmaz, M.T.; Karaman, S. Three interval thixotropy test (3ITT) in food applications: A novel technique to determine structural regeneration of mayonnaise under different shear conditions. Food Res. Int. 2015, 70, 125-133. [CrossRef]

37. Martinez, R.M.; Rosado, C.; Velasco, M.V.R.; Lannes, S.C.S.; Baby, A.R. Main features and applications of organogels in cosmetics. Int. J. Cosmet. Sci. 2019, 41, 109-117. [CrossRef]

38. Esposito, C.L.; Tardif, V.; Sarrazin, M.; Kirilov, P.; Roullin, V.G. Preparation and characterization of 12-HSA-based organogels as injectable implants for the controlled delivery of hydrophilic and lipophilic therapeutic agents. Mater. Sci. Eng. C 2020, 114, 110999. [CrossRef] [PubMed]

39. Relkin, P.; Yung, J.M.; Kalnin, D.; Ollivon, M. Structural behaviour of lipid droplets in protein-stabilized nano-emulsions and stability of $\alpha$-tocopherol. Food Biophys. 2008, 3, 163-168. [CrossRef]

40. Kamal, M.A.; Raghunathan, V.A. Modulated phases of phospholipid bilayers induced by tocopherols. Biochim. Biophys. Acta Biomembr. 2012, 1818, 2486-2493. [CrossRef] [PubMed]

41. Lv, X.; Zhang, S.; Ma, H.; Dong, P.; Ma, X.; Xu, M.; Tian, Y.; Tang, Z.; Peng, J.; Chen, H.; et al. In situ monitoring of the structural change of microemulsions in simulated gastrointestinal conditions by SAXS and FRET. Acta Pharm. Sin. B 2018, 8, 655-665. [CrossRef]

42. Takeno, H.; Mochizuki, T.; Yoshiba, K.; Kondo, S.; Dobashi, T. Self-assembling Structures and Sol-Gel Transition of Optically Active and Racemic 12-Hydroxystearic Acids in Organic Solvents. In Gels: Structures, Properties, and Functions. Progress in Colloid and Polymer Science; Tokita, M.N.K., Ed.; Springer: Berlin/Heidelberg, Germany, 2009; p. 47.

43. Blake, A.I.; Co, E.D.; Marangoni, A.G. Structure and physical properties of plant wax crystal networks and their relationship to oil binding capacity. JAOCS J. Am. Oil Chem. Soc. 2014, 91, 885-903. [CrossRef]

44. Chopin-Doroteo, M.; Morales-Rueda, J.A.; Dibildox-Alvarado, E.; Charó-Alonso, M.A.; de la Peña-Gil, A.; Toro-Vazquez, J.F. The Effect of Shearing in the Thermo-mechanical Properties of Candelilla Wax and Candelilla Wax-Tripalmitin Organogels. Food Biophys. 2011, 6, 359-376. [CrossRef]

45. Takeno, H.; Maehara, A.; Kuchiishi, M.; Yoshiba, K.; Takeshita, H.; Kondo, S.; Dobashi, T.; Takenaka, M.; Hasegawa, H. Structural and thermal properties of unpurified and purified 12-hydroxystearic acid solutions. J. Fiber Sci. Technol. 2012, 68, 248-252. [CrossRef]

46. Pedersen, J.S. Analysis of small-angle scattering data from colloids and polymer solutions: Modeling and least-squares fitting Adv. Colloid Interface Sci. 1997, 70, 171-210. [CrossRef]

47. Sundblom, A.; Oliveira, C.L.P.; Palmqvist, A.E.C.; Pedersen, J.S. Modeling in situ small-angle X-ray scattering measurements following the formation of mesostructured silica. J. Phys. Chem. C 2009, 113, 7706-7713. [CrossRef]

48. Pedersen, J.S.; Hansen, S.; Bauer, R. The aggregation behavior of zinc-free insulin studied by small-angle neutron-scattering. Eur. Biophys. J. 1994, 22, 379-389. [CrossRef]

49. Pinsky, M.A.; Karlin, S. An Introduction to Stochastic Modeling; Pinsky, M.A., Karlin, S., Eds.; Academic Press: Boston, MA, USA, 2011.

50. Kissell, R.; Poserina, J. Optimal Sports Math, Statistics, and Fantasy; Kissell, R., Poserina, J., Eds.; Academic Press: Boston, MA, USA, 2017. 
51. De, S.; Krishnan, P.; van der Schaaf, J.; Kuipers, J.A.M.; Peters, E.A.J.F.; Padding, J.T. Viscoelastic effects on residual oil distribution in flows through pillared microchannels. J. Colloid Interface Sci. 2018, 510, 262-271. [CrossRef]

52. Oliveira, C.L.P.; Behrens, M.A.; Pedersen, J.S.; Erlacher, K.; Otzen, D.; Pedersen, J.S. A SAXS Study of Glucagon Fibrillation. J. Mol. Biol. 2009, 387, 147-161. [CrossRef] 